\title{
Valoración integral de los ecosistemas cenagosos del Medio Atrato, Chocó. Caso ciénaga Grande de Beté
}

\section{Integral assessment of wetland ecosystem in the Medio Atrato, Chocó. Case ciénaga Grande Beté wetland}

\author{
Lady Vargas', Gustavo Gonzáles², Rubén Darío Sepúlveda²
}

\section{Resumen}

El aumento de los impactos derivados de las actividades antrópicas sobre los ecosistemas, ha generado una creciente preocupación sobre el uso y la disponibilidad de la base de bienes y servicios ambientales, los cuales se han convertido en el objetivo de valoración de la economía ambiental. Con el fin de valorar integralmente los bienes y servicios de la ciénaga Grande de Beté en el Medio Atrato, Chocó, se empleó la metodología de valoración contingente, a partir de la identificación del listado de los mismos, la aplicación de una encuesta y el análisis econométrico de los datos. Se identificaron 36 bienes y servicios ambientales y culturales asociados con el ecosistema y se estimó que en promedio los encuestados están dispuestos a pagar un valor de $\$ 23,644.12$ por los bienes y $\$ 28,977.41$ para el caso de los servicios ambientales. Se encontró que la disponibilidad a pagar no está asociada con los costos de uso de bienes de mercado, sino que la valoración de la ciénaga presenta una importancia global derivada de su existencia más como un recurso natural que es parte integral de sus vidas, de ahí que la disponibilidad a pagar haya sido mayor para los servicios que para los bienes ambientales y a su vez que esta haya sido positiva independientemente del estado de variables como nivel de ingresos, educación, ocupación, lugar de residencia y edad. La propuesta de valorar integralmente los bienes y servicios ecosistémicos contribuye a que las decisiones de desarrollo se ajusten de mejor forma a la realidad ambiental.

Palabras clave: Ciénaga Grande de Beté, Disponibilidad a pagar, Valoración contingente, Valoración integral.

\begin{abstract}
Increased impacts of human activities on ecosystems, has generated a growing concern about the use and availability of the basis of environmental goods and services, which have become the target valuation of environmental economics. In order fully value the goods and services of the Grande de Beté wetland in the Medio Atrato, Chocó, the contingent valuation methodology was used, from the identification listing of them, the implementation of a survey and econometric analysis data. 36 environmental and cultural goods and services associated with the ecosystem were identified and estimated that on average respondents are willing to pay a $\$ 23,644.12$ for goods and $\$ 28,977.41$ for the case of environmental services. It was found that the willingness to pay is not associated with usage costs of market goods, but the valuation of the wetland presents a more global importance derived from its existence more like a natural resource that is an integral part of their
\end{abstract}

1 Investigadora de proyectos especiales, Instituto de Investigaciones Ambientales del Pacífico (IIAP), Chocó, Quibdó. e-mail: 1vargas@iiap.org.co

2 Profesor, Universidad Pontificia Bolivariana de Montería, Córdoba, Colombia. e-mail: ruben.sepulveda@upb.edu.co gonzalez_gustavo_@hotmail.com

Recibido: 12 de noviembre de 2012 Aceptado: 23 de enero de 2013 


\section{Valoración integral de ecosistemas cenagosos. L Vargas et al.}

lives, hence the willingness to pay has been increased for services for environmental goods and in turn this has been positive regardless of the state of variables such as income, education, occupation, place of residence and age. The proposal to fully assess the ecosystem goods and services contributes to development decisions to better conform to the environmental reality.

Keywords: Ciénaga Grande de Beté, Comprehensive assessment, Contingent valuation, Willingness to pay.

\section{Introducción}

En la actualidad ha crecido la preocupación por los impactos derivados de las actividades humanas sobre los ecosistemas, debido a que el capital natural se ha reducido considerablemente por el deterioro de ecosistemas hídricos y boscosos. De ahí que la problemática ambiental actual gire entorno con el uso inadecuado de la base de bienes y servicios ambientales y su creciente degradación, lo cual ha generado una urgente necesidad de adelantar procesos de la valoración económica ambiental, con el fin de abordar esta problemática y cuantificar los costos ambientales de las actividades antrópicas, con el fin de diseñar los mecanismos de regulación e incentivos apropiados para mitigar los impactos de la sobrexplotación de los recursos naturales.

En este sentido, la economía ambiental surgió como una respuesta a la búsqueda del desarrollo sostenible o la sostenibilidad. Este tipo de economía apunta a la asignación óptima y al uso eficiente de los recursos escasos y su objetivo es encontrar el nivel óptimo de bienestar social. La economía ambiental hace énfasis en la importancia de la valoración económica, la asignación de derechos de propiedad y la incorporación de mecanismos de mercado en la regulación y gestión de los bienes públicos o "libres" que integran el medioambiente. Estos valores teóricos están apoyados en el caálculo de los “costos de oportunidad" "precios sombra" o "valores de contingencia" (Naredo 1992). De ahí surgieron las diferentes corrientes de valoración económica ambiental, las cuales constituyen un intento de asignar valores cuantitativos a los bienes y servicios proporcionados por recursos naturales independientemente de si tienen mercado, es decir, encontrar un valor económico que se aproxime a los beneficios que estos generan a la sociedad. Partiendo de estos conceptos planteados por la economía ambiental, se programó el desarrollo del proyecto de valoración integral de los ecosistemas cenagosos del municipio del Medio Atrato, caso Ciénaga Grande de Beté, en el cual se buscó identificar los bienes y servicios que presta el ecosistema a nivel económico, ambiental y cultural, seleccionar una metodología que permita el análisis integrado de los mismos y la aplicación de esta metodología, de tal manera que se pueda obtener un valor económico integral del sitio, como una herramienta para la toma de decisiones de manejo y conservación de esta área tan valiosa.

Los ecosistemas cenagosos se consideran sistemas naturales de amortiguación de inundaciones, hábitat de muchos organismos y fuente de sustento socioeconómico de las comunidades localizadas a su alrededor, particularmente la ciénaga Grande de Beté que constituye un lugar de confluencia de una gran diversidad biológica, un sistema natural que además de proteger a las comunidades de las inundaciones, les brinda el sustento diario. De allí la importancia de conocimiento y su valoración integral, como una herramienta que permita la conservación y la toma de decisiones en torno al manejo económico y cultural que se debe dar a este ecosistema. El presente trabajo tomó como base la información generada en el proyecto "Inventario, priorización y caracterización de las ciénagas del municipio del Medio Atrato, Chocó" (IIAP 2008), para identificar los bienes y servicios prestados por el ecosistema, a partir de lo cual se seleccionó una metodología de valoración ambiental que permitiera determinar el valor total del mismo basado en las preferencias y percepciones de las comunidades beneficiadas, debido a la ausencia de un mercado que indique precios y cantidades, para la mayoría de los bienes ambientales y culturales que ofrece la ciénaga.

Área de estudio. El municipio de Medio Atrato cuya extensión es de $562 \mathrm{~km}^{2}$, cuenta con 10 corregimientos, 23 veredas y 7 comunidades indígenas, entre los que se encuentran Baudogrande, Puné, Puerto Salazar, San Francisco de Tauchigadó, Boca de Agua Clara y Boca de Bebará, entre otros. Es rico por su exuberante selva tropical, diversidad de flora y fauna así como en fuentes hídricas, enmarcadas en una serie de ciénagas que han representado para las comunidades aledañas a estas una fuente de sustento diario a través del tiempo; basan su economía en actividades como la pesca, agricultura, caza, extracción de madera y minería en menor escala. Son personas de escasos recursos económicos, con alto índice de necesidades insatisfechas, carentes de asistencia social y técnica por el estado. $\mathrm{Su}$ cabecera municipal es Beté y sus principales accidentes orográficos, los constituye la Serranía del Baudó, con su máxima altura el Alto del Buey $(1.810 \mathrm{~m})$, la cual se origina en inmediaciones del río San Juan y sigue paralelamente a la costa pacífica hasta unirse con la del Darién, en el norte, delimitando al Chocó y a Colombia de Panamá. La mayor parte del territorio se halla dentro de las zonas de las calmas ecuatoriales, por lo tanto el régimen de lluvias se prolonga durante todo el año, registrándose hasta $12.000 \mathrm{~mm}^{3}$ de precipitación. La temperatura se encuentra entre $28^{\circ} \mathrm{y}$ $30^{\circ} \mathrm{C}$, con un alto grado de humedad ambiental. La ciénaga Grande de Beté se localiza en la cabecera municipal en las coordenadas $6^{\circ} 0$ ' 22.96" y $76^{\circ} \mathrm{N}$ y $46^{\prime} 11.23$ 'W, a un km 


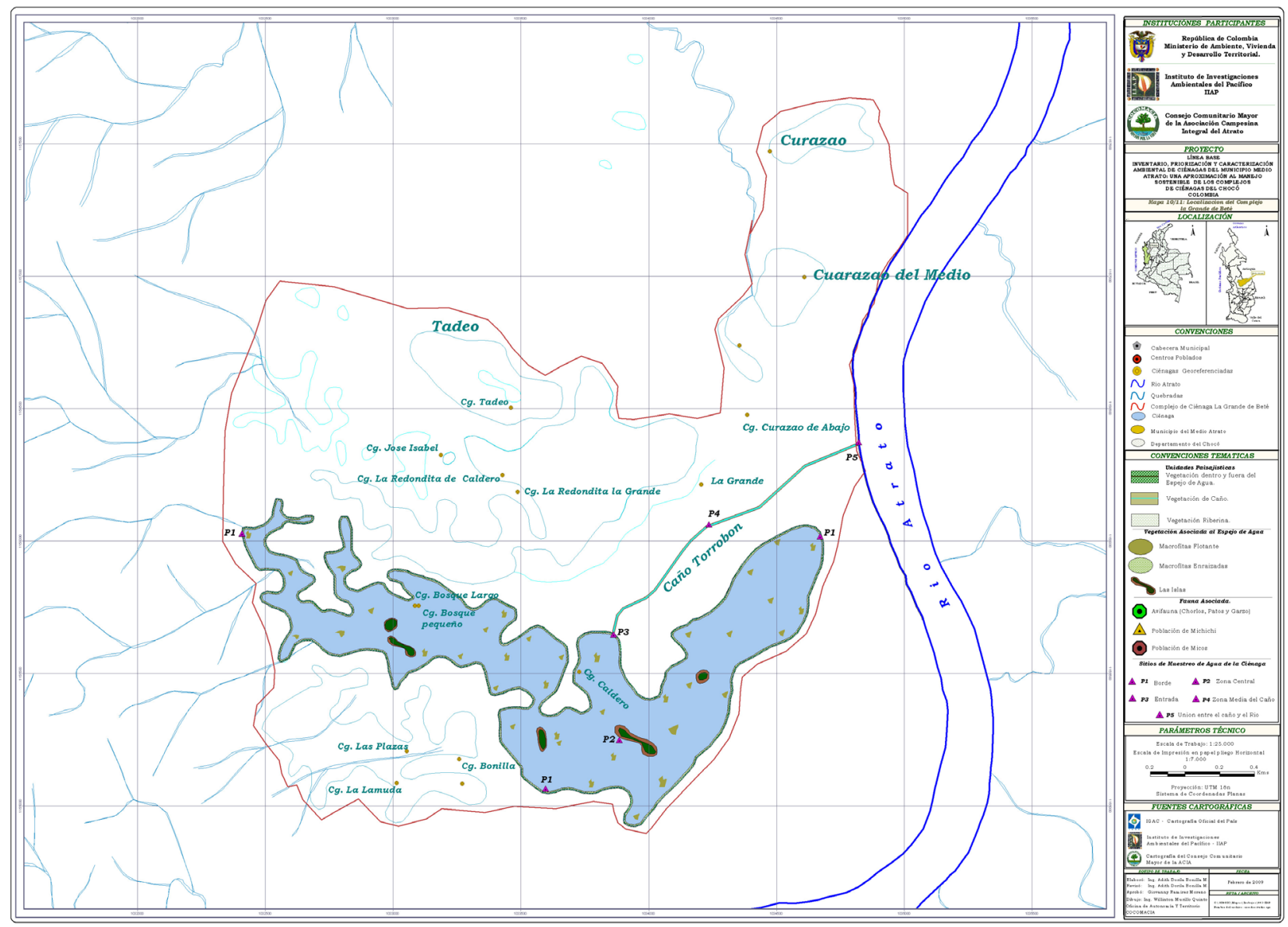

Figura 1. Localización del complejo Ciénaga Grande de Beté.

aproximadamente de la margen izquierda del río Atrato, con un área total de 168.194 ha. Es una ciénaga principal o de tipo 1, conectada de forma directa al río a través de su caño Amé (Figura 1).

\section{Métodos}

Identificación de bienes y servicios ambientales del ecosistema. Para la identificación de los bienes y servicios ambientales del complejo cenagoso la Grande de Beté, se tomó como base la información contenida en el informe final del proyecto "Inventario, priorización y caracterización de las ciénagas del municipio del Medio Atrato, Chocó", elaborado por el IIAP (2008). Se revisaron en detalle los capítulos relacionados con el componente sociocultural, económico y ambiental, lo que permitió identificar los bienes y servicios ambientales prestados por el ecosistema partiendo de la información diagnóstica levantada en campo en el proyecto mencionado, el cual incluye información biológica, física, social, ambiental, económica y análisis ecosistémicos. Se elaboró una matriz que describe los bienes y servicios identificados así como su tipología, mercado y demandantes potenciales.

Recolección de la información. Para recolectar la información se aplicó una encuesta a partir de la definición poblacional de área cercana y colindante a la ciénaga (población de Quibdó y Medio Atrato). La encuesta diseñada fue probada de manera práctica con el $10 \%$ de la población total y ajustada de acuerdo con los resultados obtenidos, antes de la aplicación definitiva (Figura 2).

Cálculos estadísticos y análisis econométricos. Se utilizó la metodología de valoración contingente para obtener el valor total del ecosistema (MAVDT 2003). Con el fin de evidenciar la capacidad explicativa de la teoría económica y la posibilidad de valorar un activo ambiental, se ajustó un modelo logit binomial con información tomada de encuestas que muestran las elecciones de uso de bienes y servicios ambientales de la ciénaga. Por una parte se estimó la disponibilidad a pagar de los individuos por el consumo de bienes ambientales y por otra parte de los servicios ambientales asociados con un conjunto de regresores explicativos. Para esto fue necesario la identificación de variables explicativas, procediendo con la estimación y validación del modelo, interpretación de los resultados y 


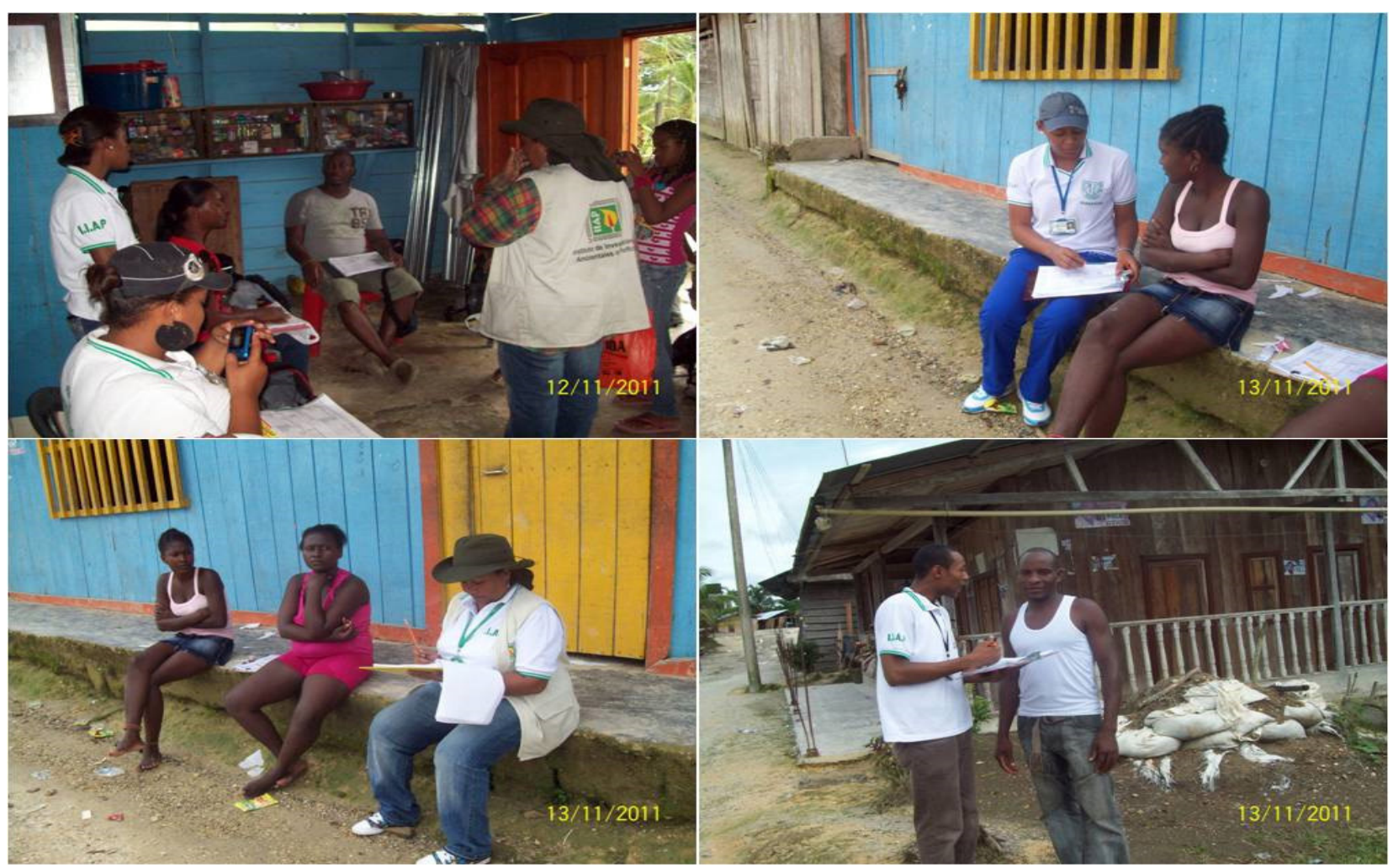

Figura 2. Aplicación de encuestas.

predicción tomando como base la información estadística disponible de la encuesta, donde se definen las variables explicativas y la variable dependiente de la especificación logit utilizada, pudiendo así analizar los resultados sobre la demanda de bienes y servicios ambientales.

De acuerdo con lo anterior, se tuvieron dos funciones logísticas para estimar: una disponibilidad a pagar referente a los bienes ambientales extraídos en la ciénaga y otra mostrando la disponibilidad a pagar por servicios ambientales, explicados por la utilización de regresores con condición de extracción de la ciénaga, valores de disponibilidad a pagar y condición socioeconómica de los individuos encuestados.

Variable dependiente. Modelo de bienes (Var2a). Variable binaria que muestra disponibilidad a pagar por cambios en el bienestar por el uso de bienes ambientales. Toma el valor de uno si los individuos determinan un cambio en bienestar positivo; toma el valor de cero si tienen su consumo un cambio significativo en su bienestar.

Modelo de servicios (Var6a). Variable binaria que muestra disponibilidad a pagar por cambios en el bienestar por el uso de servicios ambientales. Toma el valor de uno si los individuos determinan un cambio en bienestar positivo; toma el valor de cero si tiene su consumo un cambio significativo en su bienestar.

\section{Variables independientes}

Modelo de bienes ambientales
Var1: Valor monetario de los gastos en bienes ambientales extraídos por un consumidor en la ciénaga de Beté. Var3: Valor monetario del encuestado de la disponibilidad a pagar anual por perdurar la ciénaga de Beté. Var4: Valor de horas de voluntariado anuales que mostraría un encuestado por mantener la ciénaga de Beté. Var9a: Variable binaria con valor en uno si la ciénaga contribuye en prácticas culturales.

Var9b: Variable binaria con valor en uno si la ciénaga contribuye en la conservación de prácticas productivas. Var9c: Variable binaria con valor en uno si la ciénaga contribuye en la conservación de prácticas culturales. Var9d: Variable binaria con valor en uno si la ciénaga contribuye en el fortalecimiento de la identidad cultural. Var9e: Variable binaria con valor en uno si la ciénaga contribuye en la protección del conocimiento tradicional. Sexo: Variable binaria con valor en uno si es hombreIngresos: Valor monetario de los ingresos mensuales del encuestado.

Energía: Valor monetario del pago energía mensual de los servicios públicos.

Edad: En años.

Escolaridad: Como último nivel alcanzado en años. Ocupación: Variable binaria que tiene valor de uno con ocupación productiva en relación directa con la ciénaga. Modelo de servicios ambientales 


\section{Bioetnia Volumen 10, 2013}

- Var5: Valor monetario de los gastos en servicios ambientales extraídos por un consumidor en la ciénaga de Beté.

- Var7: Valor monetario del encuestado de la disponibilidad a pagar anual por perdurar la ciénaga de Beté.

Var8: Valor de horas de voluntariado anuales que mostraría un encuestado por mantener la ciénaga de Beté. Var9a: Variable binaria con valor en uno si la ciénaga contribuye en prácticas culturales.

- Var9b: Variable binaria con valor en uno si la ciénaga contribuye en la conservación de prácticas productivas.

- Var9c: Variable binaria con valor en uno si la ciénaga contribuye en la conservación de prácticas culturales.

- Var9d: Variable binaria con valor en uno si la ciénaga contribuye en el fortalecimiento de la identidad cultural.

- Var9e: Variable binaria con valor en uno si la ciénaga contribuye en la protección del conocimiento tradicional. Sexo: Variable binaria con valor en uno si es hombre.

- Ingresos: Valor monetario de los ingresos mensuales del encuestado.

- Energía: Valor monetario del pago energía mensual de los servicios públicos.

Edad: En años.

- Escolaridad: Como último nivel alcanzado en años.

- Ocupación: Variable binaria que tiene valor de uno con ocupación productiva en relación directa con la ciénaga.

Análisis econométrico de la elección de alternativas.

Para el análisis econométrico se partió del planteamiento que se pretende explicar la elección hecha por el decisor, como función de variables que lo caracterizan y que se denota por regresores, añadiendo un término de error que explique las diferencias entre los valores observados de Pi (uno o cero) y sus valores previstos. En el modelo se define $\mathrm{Pi}=($ muestra disponibilidad a pagar $=1)$ si el consumidor tiene un cambio de bienestar por los consumo de bienes o servicios, $1-\mathrm{Pi}=0$ (no muestra disponibilidad a pagar $=0$ ) si el consumidor no tiene un cambio de bienestar por los consumo de bienes o servicios. La especificación funcional del modelo de demanda de bienes ambientales utilizado bajo condiciones de probabilidad y significancia de los regresores es el siguiente:

Para el modelo de bienes ambientales:

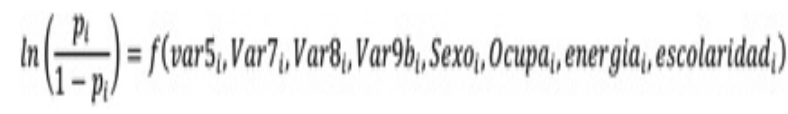

donde:

$\mathbf{p}=1 \mathrm{Si}$ determina una disponibilidad a pagar por mejora de bienestar.

$\mathbf{1 - p}=$ En otro caso.

- Var1: Valor monetario de los gastos en bienes ambientales extraídos por un consumidor en la ciénaga de Beté. Var3: Valor monetario del encuestado de la disponibi- lidad a pagar anual por perdurar la ciénaga de Beté.

Var4: Valor de horas de voluntariado anuales que mostraría un encuestado por mantener la ciénaga de Beté.

Var9e: Variable binaria con valor en uno si la ciénaga contribuye en la protección del conocimiento tradicional. Sexo: Variable binaria con valor en uno si es hombre.

Ingresos: Valor monetario de los ingresos mensuales del encuestado.

Energía: Valor monetario del pago energía mensual de los servicios públicos.

Edad: En años.

Escolaridad: Como último nivel alcanzado en años.

Ocupación: Variable binaria que tiene valor de uno con ocupación productiva con relación directa con la ciénaga.

Para el modelo de servicios ambientales:

$\ln \left(\frac{p_{i}}{1-p_{i}}\right)$

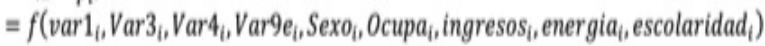

donde:

$\mathbf{p}=1$ Si determina una disponibilidad a pagar por mejora de bienestar.

1-p=En otro caso.

Var5: Valor monetario de los gastos en bienes ambientales extraídos por un consumidor en la ciénaga de Beté.

- Var7: Valor monetario del encuestado de la disponibilidad a pagar anual por perdurar la ciénaga de Beté.

- Var8: Valor de horas de voluntariado anuales que mostraría un encuestado por mantener la ciénaga de Beté.

Var9b: Variable binaria con valor en uno si la ciénaga contribuye en la conservación de prácticas productivas. Sexo: Variable binaria con valor en uno si es hombre.

- Energía: Valor monetario del pago energía mensual de los servicios públicos.

. Edad: En años.

- Escolaridad: Como último nivel alcanzado en años.

- Ocupación: Variable binaria que tiene valor de uno con ocupación productiva con relación directa con la ciénaga.

Formalizando en forma lineal, con una variable latente para bienes ambientales:

$\begin{aligned} \ln \left(\frac{\mathrm{P}_{i}}{1-\mathrm{P}_{1}}\right)= & \beta_{1}+\beta_{2} * \operatorname{Var}_{i}+\beta_{3} * \operatorname{Var}_{i}+\beta 4 * \operatorname{Var}_{i}+\beta_{5} * \operatorname{Var}_{e_{i}}+\beta_{6} * \text { Sexo }_{i} \\ & +\beta_{7} * \text { Ingreso }_{i}+\beta_{8} * \text { Energia }_{i}+\beta_{9} * \text { Escolaridad }_{i}+\mu_{i}\end{aligned}$

Formalizando en forma lineal, con una variable latente para servicios ambientales:

$$
\begin{aligned}
\ln \left(\frac{P_{i}}{1-P_{i}}\right)= & \beta_{1}+\beta_{2} * \operatorname{Var} 5_{i}+\beta_{3} * \operatorname{Var}_{i}+\beta 4 * \operatorname{Var} \beta_{i}+\beta_{5} * \operatorname{Var} 9 b_{i}+\beta_{6} * \operatorname{Sexo}_{i} \\
& +\beta_{7} * \text { Energia }_{i}+\beta_{8} * \text { Escolaridad }_{i}+\mu_{i}
\end{aligned}
$$


Valoración integral de ecosistemas cenagosos. L Vargas et al.

Tabla 1

Matriz de bienes y servicios ambientales, económicos y culturales prestados por la ciénaga Grande de Beté

\begin{tabular}{|c|c|c|c|c|c|c|c|c|}
\hline \multicolumn{3}{|c|}{ Bienes ambientales } & \multicolumn{3}{|c|}{ Servicios ambientales } & \multicolumn{3}{|c|}{ Servicios culturales } \\
\hline Bien & Tipo & $\begin{array}{c}\text { Mercado/ } \\
\text { demandantes } \\
\text { potenciales }\end{array}$ & Bien/servicio & Tipo & $\begin{array}{l}\text { Mercado/ } \\
\text { demandantes } \\
\text { potenciales }\end{array}$ & Bien/ servicio & Tipo & $\begin{array}{l}\text { Mercado/ } \\
\text { demandantes } \\
\text { potenciales }\end{array}$ \\
\hline $\begin{array}{l}\text { Madera/ } \\
\text { Especies } \\
\text { forestales } \\
\text { para extracción } \\
\text { de maderas }\end{array}$ & $\begin{array}{l}\text { Uso } \\
\text { directo }\end{array}$ & $\begin{array}{l}\text { Sí/mercado } \\
\text { de madera } \\
\text { regional y } \\
\text { nacional }\end{array}$ & $\begin{array}{l}\text { Control } \\
\text { de erosión }\end{array}$ & $\begin{array}{l}\text { Uso } \\
\text { indirecto }\end{array}$ & $\begin{array}{l}\text { No/poblaciones } \\
\text { locales }\end{array}$ & $\begin{array}{l}\text { Conservación } \\
\text { de técnicas } \\
\text { tradicionales } \\
\text { de producción }\end{array}$ & $\begin{array}{l}\text { Valor de } \\
\text { existencia }\end{array}$ & $\begin{array}{l}\text { No/poblaciones } \\
\text { locales }\end{array}$ \\
\hline $\begin{array}{l}\text { Especies } \\
\text { vegetales } \\
\text { para uso } \\
\text { medicinal }\end{array}$ & $\begin{array}{l}\text { Uso } \\
\text { directo }\end{array}$ & $\begin{array}{l}\text { Sí/mercado } \\
\text { agrícola } \\
\text { regional } \\
\text { (Quibdó y } \\
\text { otro municipios) }\end{array}$ & $\begin{array}{l}\text { Control de } \\
\text { inundaciones }\end{array}$ & $\begin{array}{l}\text { Uso } \\
\text { indirecto }\end{array}$ & $\begin{array}{l}\text { No/poblaciones } \\
\text { locales }\end{array}$ & $\begin{array}{l}\text { Conservación } \\
\text { de prácticas } \\
\text { culturales }\end{array}$ & $\begin{array}{l}\text { Valor de } \\
\text { existencia }\end{array}$ & $\begin{array}{l}\text { No/poblaciones } \\
\text { locales }\end{array}$ \\
\hline Agua & $\begin{array}{l}\text { Uso } \\
\text { directo }\end{array}$ & $\begin{array}{l}\text { Sí/mercado } \\
\text { agrícola } \\
\text { regional } \\
\text { (Quibdó y otro } \\
\text { municipios) }\end{array}$ & $\begin{array}{l}\text { Fijación y } \\
\text { reciclaje de } \\
\text { nutrientes }\end{array}$ & $\begin{array}{l}\text { Uso } \\
\text { indirecto }\end{array}$ & $\begin{array}{l}\text { No/poblaciones } \\
\text { locales }\end{array}$ & $\begin{array}{l}\text { Fortalecimiento } \\
\text { de la identidad } \\
\text { cultural }\end{array}$ & $\begin{array}{l}\text { Valor de } \\
\text { existencia }\end{array}$ & $\begin{array}{l}\text { No/poblaciones } \\
\text { locales }\end{array}$ \\
\hline Semillas & $\begin{array}{l}\text { Uso } \\
\text { directo }\end{array}$ & $\begin{array}{l}\text { Sí/mercado) } \\
\text { agrícola } \\
\text { regional } \\
\text { (Quibdó y otro } \\
\text { municipios }\end{array}$ & $\begin{array}{l}\text { Protección } \\
\text { y formación } \\
\text { de suelos }\end{array}$ & $\begin{array}{l}\text { Uso } \\
\text { directo }\end{array}$ & $\begin{array}{l}\text { No/poblaciones } \\
\text { locales }\end{array}$ & $\begin{array}{l}\text { Protección del } \\
\text { conocimiento } \\
\text { tradicional }\end{array}$ & $\begin{array}{l}\text { Valor de } \\
\text { existencia }\end{array}$ & $\begin{array}{l}\text { No/poblaciones } \\
\text { locales }\end{array}$ \\
\hline $\begin{array}{l}\text { Especies } \\
\text { vegetales } \\
\text { y frutos } \\
\text { comestibles }\end{array}$ & $\begin{array}{l}\text { Uso } \\
\text { directo }\end{array}$ & $\begin{array}{l}\text { Sí/mercado } \\
\text { agrícola } \\
\text { regional } \\
\text { (Quibdó y otro } \\
\text { municipios) }\end{array}$ & $\begin{array}{l}\text { Retención } \\
\text { de sedimentos }\end{array}$ & $\begin{array}{l}\text { Uso } \\
\text { indirecto }\end{array}$ & $\begin{array}{l}\text { No/poblaciones } \\
\text { ocales }\end{array}$ & & & \\
\hline $\begin{array}{l}\text { Recursos no } \\
\text { maderables del } \\
\text { bosque (resinas } \\
\text { y látex) }\end{array}$ & $\begin{array}{l}\text { Uso } \\
\text { directo }\end{array}$ & $\begin{array}{l}\text { No/industria } \\
\text { regional y } \\
\text { nacional }\end{array}$ & $\begin{array}{l}\text { Captura de } \\
\text { carbono }(\mathrm{CO} 2)\end{array}$ & $\begin{array}{l}\text { Uso } \\
\text { directo }\end{array}$ & $\begin{array}{l}\text { No/poblaciones } \\
\text { locales }\end{array}$ & & & \\
\hline $\begin{array}{l}\text { Recursos no } \\
\text { maderables del } \\
\text { bosque } \\
\text { (colorantes) }\end{array}$ & $\begin{array}{l}\text { Uso } \\
\text { directo }\end{array}$ & $\begin{array}{l}\text { No/industria } \\
\text { regional y } \\
\text { nacional }\end{array}$ & $\begin{array}{l}\text { Regulación } \\
\text { del clima }\end{array}$ & $\begin{array}{l}\text { Uso } \\
\text { indirecto }\end{array}$ & $\begin{array}{l}\text { No/poblaciones } \\
\text { locales }\end{array}$ & & & \\
\hline $\begin{array}{l}\text { Recursos no } \\
\text { maderables del } \\
\text { bosque } \\
\text { (venenos) }\end{array}$ & $\begin{array}{l}\text { Uso } \\
\text { directo }\end{array}$ & $\begin{array}{l}\text { No/industria } \\
\text { regional y } \\
\text { nacional }\end{array}$ & Etnoeducación & $\begin{array}{l}\text { Uso } \\
\text { directo }\end{array}$ & $\begin{array}{l}\text { Sí/poblaciones } \\
\text { locales }\end{array}$ & & & \\
\hline $\begin{array}{l}\text { Fauna } \\
\text { acuática }\end{array}$ & $\begin{array}{l}\text { Uso } \\
\text { directo }\end{array}$ & $\begin{array}{l}\text { Sí/mercado } \\
\text { regional } \\
\text { (Quibdó y otro } \\
\text { municipios) }\end{array}$ & Investigación & $\begin{array}{l}\text { Uso } \\
\text { directo }\end{array}$ & $\begin{array}{l}\text { Sí/poblaciones } \\
\text { locales, global }\end{array}$ & & & \\
\hline $\begin{array}{l}\text { Fauna } \\
\text { terrestre }\end{array}$ & $\begin{array}{l}\text { Uso } \\
\text { directo }\end{array}$ & $\begin{array}{l}\text { Sí/mercado } \\
\text { local y } \\
\text { alrededores }\end{array}$ & $\begin{array}{l}\text { Recreación } \\
\text { y turismo }\end{array}$ & $\begin{array}{l}\text { Uso } \\
\text { directo }\end{array}$ & $\begin{array}{l}\text { No/poblaciones } \\
\text { locales, } \\
\text { regionales, } \\
\text { nacionales }\end{array}$ & & & \\
\hline Aves & $\begin{array}{l}\text { Uso } \\
\text { directo }\end{array}$ & $\begin{array}{l}\text { Sí/mercado } \\
\text { local y } \\
\text { alrededores }\end{array}$ & Movilidad & $\begin{array}{l}\text { Uso } \\
\text { directo }\end{array}$ & $\begin{array}{l}\text { Sí/poblaciones } \\
\text { locales, } \\
\text { regionales }\end{array}$ & & & \\
\hline $\begin{array}{l}\text { Recursos no } \\
\text { maderables } \\
\text { del bosque } \\
\text { (fibras) }\end{array}$ & $\begin{array}{l}\text { Uso } \\
\text { directo }\end{array}$ & $\begin{array}{l}\text { No/industria } \\
\text { regional y } \\
\text { nacional }\end{array}$ & $\begin{array}{l}\text { Protección } \\
\text { de la cuenca } \\
\text { del río Atrato }\end{array}$ & $\begin{array}{l}\text { Uso } \\
\text { indirecto }\end{array}$ & $\begin{array}{l}\text { No/poblaciones } \\
\text { locales, } \\
\text { regionales, } \\
\text { global }\end{array}$ & & & \\
\hline $\begin{array}{l}\text { Especies } \\
\text { vegetales } \\
\text { aromáticas }\end{array}$ & $\begin{array}{l}\text { Uso } \\
\text { directo }\end{array}$ & $\begin{array}{l}\text { Sí/mercado } \\
\text { agrícola regional } \\
\text { Quibdó y otro } \\
\text { municipios) }\end{array}$ & $\begin{array}{l}\text { Hospedaje } \\
\text { de aves } \\
\text { migratorias }\end{array}$ & $\begin{array}{l}\text { Valor de } \\
\text { opción }\end{array}$ & $\begin{array}{l}\text { No/poblaciones } \\
\text { locales, } \\
\text { regionales, } \\
\text { global }\end{array}$ & & & \\
\hline $\begin{array}{l}\text { Potencial } \\
\text { genético }\end{array}$ & $\begin{array}{l}\text { Valor de } \\
\text { opción }\end{array}$ & $\begin{array}{l}\text { No/poblaciones } \\
\text { locales, } \\
\text { regionales, } \\
\text { global }\end{array}$ & $\begin{array}{l}\text { Hábitat de } \\
\text { biodiversidad } \\
\text { de interés } \\
\text { ecológico }\end{array}$ & $\begin{array}{l}\text { Valor de } \\
\text { opción }\end{array}$ & $\begin{array}{l}\text { No/poblaciones } \\
\text { locales, } \\
\text { regionales, } \\
\text { global }\end{array}$ & & & \\
\hline $\begin{array}{l}\text { Recursos no } \\
\text { maderables } \\
\text { bosque del } \\
\text { (aceites) }\end{array}$ & $\begin{array}{l}\text { Uso } \\
\text { directo }\end{array}$ & $\begin{array}{l}\text { Sí/población } \\
\text { local }\end{array}$ & $\begin{array}{l}\text { Conservación } \\
\text { de especies } \\
\text { únicas }\end{array}$ & $\begin{array}{l}\text { Valor de } \\
\text { opción }\end{array}$ & $\begin{array}{l}\text { No/poblaciones } \\
\text { locales, } \\
\text { regionales, } \\
\text { global }\end{array}$ & & & \\
\hline $\begin{array}{l}\text { Especies } \\
\text { vegetales } \\
\text { magicoreligiosas }\end{array}$ & $\begin{array}{l}\text { Uso } \\
\text { directo }\end{array}$ & $\begin{array}{l}\text { Sí/mercado } \\
\text { local y regional } \\
\text { (Quibdó y } \\
\text { otro municipios) }\end{array}$ & $\begin{array}{l}\text { Movilidad y } \\
\text { conectividad } \\
\text { de especies }\end{array}$ & $\begin{array}{l}\text { Valor de } \\
\text { opción }\end{array}$ & $\begin{array}{l}\text { No/poblaciones } \\
\text { locales, } \\
\text { regionales, } \\
\text { global }\end{array}$ & & & \\
\hline
\end{tabular}




\section{Bioetnia Volumen 10, 2013}

\section{Resultados y discusión}

Identificación de bienes y servicios ambientales. Como resultado del análisis de la información base sobre la ciénaga Grande de Beté, la cual incluyó aspectos socioculturales, ambientales y económicos, se obtuvo una matriz con 36 bienes y servicios ambientales, a partir de los cuales se realizó la valoración integral de la misma (Tabla 1).

Valoración integral de la ciénaga Grande de Beté. Los resultados de la estimación de los modelos logit binomial de elección que muestra disponibilidad a pagar por bienes o servicios ambientales permitieron analizar la influencia de las variables explicativas en la elección de la comunidad. Para el caso de los bienes ambientales (Var2a) se puede observar la importancia que tienen los parámetros que acompañan a las variables disponibilidad a pagar (var3) y horas de voluntariado (Var4), siendo las que hace el mayor aporte en significancia individual sobre la decisión del consumidor en tener disponibilidad a pagar (Tabla 2).

De acuerdo con los resultados de la Tabla 2, los valores positivos de los estimadores de los parámetros indican favorabilidad a la elección de disponibilidad a pagar. El coeficiente estimado asociado con la variable precio de mercado de bienes de consumo (Var1) no es significativo a la hora elegir una disponibilidad a pagar, por lo que el concepto de esta disponibilidad para los encuestados no está relacionado con el costo de uso de bienes de mercado, sino con una valoración de uso de bienes más integral, teniendo en cuenta que la relación obtenida entre la ciénaga y los bienes de mercado que vienen de la extracción de la misma no es significativa. Esta consideración es válida porque los encuestados asignan un mayor valor a la existencia de la ciénaga como un recurso natural que es parte integral de sus vidas (valor de existencia), más que como un valor de uso directo por el conjunto de bienes y servicios que oferta actualmente.

De otro lado, los regresores que aparecen como significativos, son los estimadores que acompañan los ingresos, el pago de energía, la escolaridad y la variable asociada con la protección del conocimiento tradicional (Var9e), lo que indica que estos aspectos presentan relevancia en la decisión de los encuestados. La significancia global presenta valores por encima de los promedios de estimación de modelos que utilizan información de corte transversal, teniendo en cuenta los valores de la prueba $\mathrm{Chi}^{2}$ de significancia global (muestra significancia del modelo a nivel general, con un resultado de un valor calculado de 25 , con un p de $0 \%$ ). También existe ajuste a los errores estándar dada la condición natural de heterocedasticidad de los datos transversales (corrección de las varianzas bajo el supuesto de White (1984), para tener más certeza en la inferencia sobre los estimadores (Tabla 3 ).

Los resultados de la estimación de la Tabla 3 muestran la disponibilidad a pagar por servicios ambientales y permiten analizar la influencia de las variables explicativas en la elección de los encuestados. La variable dependiente

Tabla 2

Cálculos en STATA 10 para bienes ambientales

\begin{tabular}{|c|c|c|c|c|c|c|}
\hline \multirow[t]{3}{*}{ Logistic regression } & & Number of obs & \multicolumn{2}{|l|}{379} & & \\
\hline & & Wald $\operatorname{chi}^{2}(9)$ & \multicolumn{2}{|l|}{25.05} & & \\
\hline & & Prob > chi ${ }^{2}$ & \multicolumn{2}{|l|}{0.0029} & & \\
\hline Log pseudolikelihood & -42.123723 & Pseudo $\mathrm{R}^{2}$ & \multicolumn{2}{|l|}{0.814} & & \\
\hline \multicolumn{7}{|c|}{ Robust } \\
\hline Var2a & Coef. & Std. Err. & $\mathbf{Z}$ & $p>z$ & \multicolumn{2}{|c|}{ [95\% Conf. Interval] } \\
\hline Var1 & $-2.51 \mathrm{E}-07$ & 5.05E-07 & -0.5 & 0.619 & $-1.24 \mathrm{E}-06$ & 7.39E-07 \\
\hline Var3 & 0.0002313 & 0.0000657 & 3.52 & 0 & 0.0001024 & 0.0003601 \\
\hline Var4 & -0.0000185 & 5.56E-06 & -3.32 & 0.001 & -0.0000294 & $-7.59 \mathrm{E}-06$ \\
\hline Var9e & 1.546404 & 0.7299768 & 2.12 & 0.034 & 0.1156758 & 2.977132 \\
\hline Sexo & -0.9407542 & 0.5115538 & -1.84 & 0.066 & -1.943381 & 0.0618728 \\
\hline Ocupación & -2.253142 & 1.244309 & -1.81 & 0.07 & -4.691944 & 0.1856594 \\
\hline Ingresos & 1.04E-06 & 3.91E-07 & 2.65 & 0.008 & 2.71E-07 & 1.80E-06 \\
\hline Energía & -0.000016 & 6.86E-06 & -2.33 & 0.02 & -0.0000294 & $-2.53 E-06$ \\
\hline Escolaridad & 0.1341684 & 0.0661822 & 2.03 & 0.043 & 0.0044537 & 0.2638832 \\
\hline _cons & -4.426313 & 1.224774 & -3.61 & 0 & -6.826825 & -2.0258 \\
\hline
\end{tabular}


Valoración integral de ecosistemas cenagosos. L Vargas et al.

Tabla 3

Cálculos con STATA 10 para servicios ambientales

\begin{tabular}{|c|c|c|c|c|c|c|}
\hline \multirow[t]{3}{*}{ Logistic regression } & & \multicolumn{2}{|c|}{ Number of obs } & 377 & & \\
\hline & & Wald $\operatorname{chi}^{2}(9)$ & \multicolumn{2}{|c|}{96.27} & & \\
\hline & & Prob $>$ chi $^{2}$ & \multicolumn{2}{|c|}{0} & & \\
\hline Log pseudolikelihood & -62.621892 & Pseudo $\mathrm{R}^{2}$ & \multicolumn{2}{|c|}{0.7011} & & \\
\hline \multicolumn{7}{|c|}{ Robust } \\
\hline Var2a & Coef. & Std. Err. & $\mathbf{Z}$ & $p>z$ & \multicolumn{2}{|c|}{ [95\% Conf. Interval] } \\
\hline Var5 & $-1.23 \mathrm{E}-06$ & $1.45 \mathrm{E}-06$ & -0.85 & 0.394 & $-4.07 \mathrm{E}-06$ & $1.60 \mathrm{E}-06$ \\
\hline Var7 & 0.0001568 & 0.0000324 & 4.83 & 0 & 0.0000932 & 0.0002204 \\
\hline Var8 & $-7.75 E-06$ & 1.92E-06 & -4.03 & 0 & -0.0000115 & $-3.99 E-06$ \\
\hline Var9b & 0.8530337 & 0.5880473 & 1.45 & 0.147 & -0.2995179 & 2.005585 \\
\hline Sexo & -0.4398458 & 0.4705077 & -0.93 & 0.35 & -1.362024 & 0.4823322 \\
\hline Ocupa & 0.4916231 & 0.4165046 & 1.18 & 0.238 & -0.3247108 & 1.307957 \\
\hline Energía & 4.86E-06 & 4.30E-06 & 1.13 & 0.258 & $-3.57 E-06$ & 0.0000133 \\
\hline Edad & -0.0205241 & 0.0175068 & -1.17 & 0.241 & -0.0548368 & 0.0137886 \\
\hline Escolaridad & -0.0149616 & 0.0579122 & -0.26 & 0.796 & -0.1284674 & 0.0985442 \\
\hline cons & -2.428558 & 1.118075 & -2.17 & 0.03 & -4.619944 & -0.2371712 \\
\hline
\end{tabular}

(Var6a) puede mostrar la importancia que tienen los parámetros que acompañan a las variables disponibilidad a pagar (Var7) y horas de voluntariado (Var8). El coeficiente estimado asociado con la variable precio de mercado de servicios ambientales de consumo (Var5) no es significativo a la hora de elegir una disponibilidad a pagar, por lo que se deduce que existe una connotación de disponibilidad a pagar por encima de los precios de mercado de los servicios que ofrece la ciénaga o ecosistema. Los regresores adicionales aparecen como no significativos, por lo que el análisis económico sería inconsistente con la estadística de valoración de la muestra. Pero la significancia global a diferencia de la individual tiene buenos resultados, al punto que están por encima de los promedios de estimación de modelos que utilizan información de corte transversal, teniendo valores de la prueba $\mathrm{Chi}^{2}$ de significancia global (muestra significancia del modelo a nivel general, con un resultado de un valor calculado de 93 , con un p de $0 \%$ ).

Cálculo de la disponibilidad a pagar. Con los resultados de la variable dependiente estimada (en este caso la probabilidad a tener disponibilidad a pagar por bienes ambientales) a través de variables monetarias (Var3 y Var4 para bienes ambientales y Var7 y Var8 para servicios ambientales), se obtuvo de acuerdo con el modelo de bienes ambientales, que en promedio los encuestados están dispuestos a pagar un valor de $\$ 23,644.12$ y $\$ 28,977.41$ para el caso de los servicios ambientales, siendo valores validados por buenos resultados econométricos, sobre todo por la significancia global. Aunque estos valores, no constituyen el precio de los recursos naturales que alberga el ecosistema cenagoso estudiado, si expresa una aproximación a la percepción comunitaria sobre la importancia y cuantificación del costo global del ecosistema en el marco de las condiciones actuales del territorio. A este respecto autores como Cruz (2006), Martínez-Alier y Roca (2000) y Castiblanco (2007) han argumentado el problema que presentan la mayoría de los métodos de valoración de bienes y servicios ecosistémicos, en cuanto a su comparabilidad y a las unidades de medida utilizadas para estos. Los niveles de comparabilidad y de conmensurabilidad de los mismos, pueden generar una pluralidad incalculable de valores dentro de la naturaleza, e incluso, elementos de esta que no pueden ser medidos, mucho menos en términos monetarios. Teniendo en cuenta estas limitantes mencionadas, se ha reconocido como una conclusión básica dentro de los postulados de la economía ecológica, la imposibilidad de una conmensurabilidad del valor total de la naturaleza tal como lo plantea la economía ambiental, aún sin tener en cuenta el valor subjetivo del placer de vivir de cada uno de los miembros de la población y que se relaciona directa e indirectamente con la naturaleza (Gómez-Baggethun y de Groot, 2007).

Interpretación descriptiva de la información. La Tabla 4 muestra que todas las variables seleccionadas tienen una variación monetaria. Se evidencia para la información 
Bioetnia Volumen 10, 2013
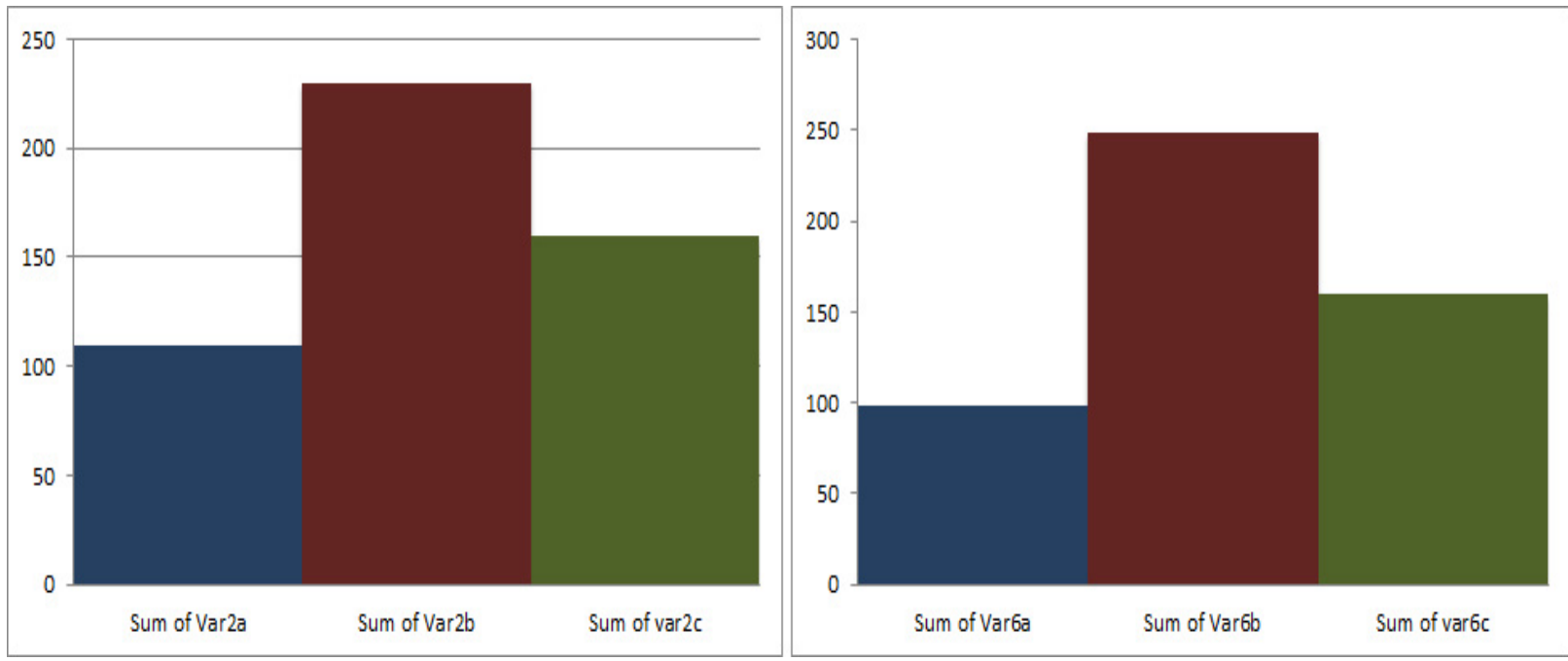

Figura 3. Tipo de disponibilidad a pagar por el consumo de bienes y servicios ambientales en la Ciénaga de Beté.

Donde:

Sum of Var2a: Elección de disponibilidad a pagar por pago monetario de bienes Sum of Var2b: Elección de disponibilidad a pagar horas de voluntariado de bienes Sum of Var2c: Elección de disponibilidad a pagar por cambio cultural de bienes Sum of Var6a: Elección de disponibilidad a pagar por pago monetario de servicios Sum of Var6b: Elección de disponibilidad a pagar horas de voluntariado de servicios Sum of Var6c: Elección de disponibilidad a pagar por cambio cultural de servicios

de condición socioeconómica cierta homogeneidad en los datos, porque el valor del coeficiente de variación es inferior a uno, seguido de la variable edad y escolaridad de los encuestados. Mientras que los ingresos y pago de energía presentan un nivel de heterogeneidad representativo, lo cual determina la capacidad explicativa de un modelo econométrico. Por el lado de las variables ambientales que se monetizaron, solo el valor de voluntariado para bienes y para servicios presentan estabilidad en variación, las demás presentaron un nivel de variabilidad importante. Estos resultados de las variables de carácter pecuniario, determinan que no es posible identificar individuos característicos con la información descriptiva, porque las medias calculadas no tienen capacidad de representación muestral. A pesar de la incapacidad de la media de representar a las variables, se puede observar la consistencia de los resultados, comparados con encuestas aplicadas por el DANE (2013), la cual tiene resultados similares en relación con el valor medio de los ingresos, su cercanía al salario mínimo (\$589.500) con un valor un poco por encima, ha sido la constante de cálculo de los valores medios de los instrumentos de recolección de información a nivel laboral.

Respecto a la escolaridad, el resultado es muy similar a lo planteado por el DANE en sus consolidados nacionales
(9.3 años), solo está un poco por debajo, lo cual es consistente, dada las condiciones de la región en términos de desarrollo. Por otro lado, la edad con un valor medio de 38 años, determina un valor que se podría asociar con un encuestado con capacidad de pago, por el rango de edad productiva que representa. Finalmente, el resultado del pago de energía presenta un nivel de variabilidad similar a los ingresos, lo cual indica que la capacidad de compra o gasto medido por los ingresos sí podría cumplir esta tarea en la investigación, porque la variable de control (en este caso el pago de energía) refleja su condición de variación en forma similar.

En el caso de las variables de modelación ambiental que se monetizaron, vale la pena mencionar que los valores de los servicios ambientales tienen un precio medio siempre por encima de los bienes ambientales, lo que puede ser un indicio de una mayor percepción de importancia de los servicios ambientales frente a los bienes ambientales. En este caso la mayor parte de los encuestados son hombres (anotar cuántos), lo que evidencia posiblemente que este género representa la cabeza de hogar en la mayoría de los casos y por tanto tiene una mayor capacidad de compra.

Para la valoración de la ciénaga se categorizó a la ocupación de acuerdo con la capacidad de relacionarse 
Valoración integral de ecosistemas cenagosos. L Vargas et al.

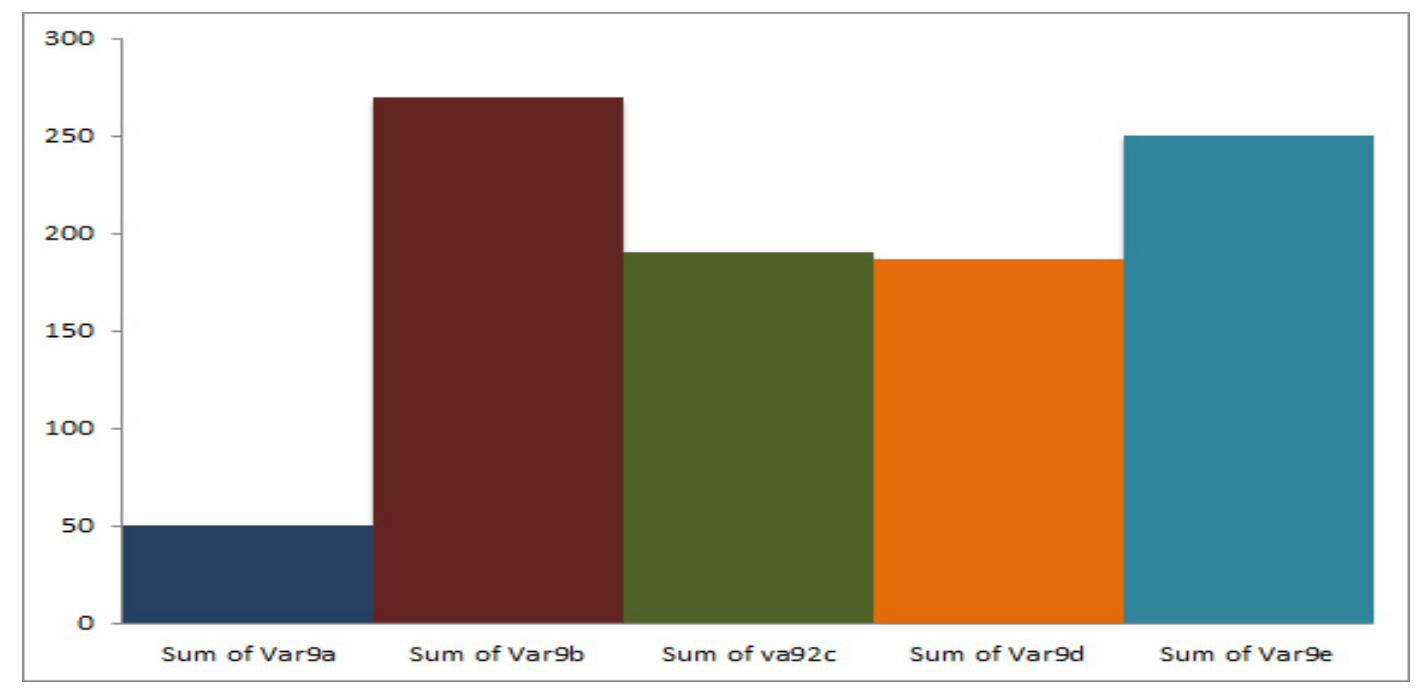

Figura 4. Elección de los encuestados frente a la contribución de la Ciénaga de Beté prácticas culturales.

Donde:

Sum of Var9a: Elección de prácticas culturales

Sum of Var9b: Elección de conservación de prácticas productivas

Sum of Var9c: Elección de conservación de prácticas culturales

Sum of Var9d: Elección de fortalecimiento de la identidad cultural

Sum of Var9e: Elección de protección de conocimiento tradicional

al individuo con el activo ambiental, en este sentido el mayor peso en ocupaciones no tienen relación directa con la ciénaga, de ahí que los valores de opción y existencia hayan sido más representativos en la disponibilidad a pagar.

La Figura 3 muestra que el aporte en horas de voluntariado presenta mayor frecuencia de elecciones bien sea para bienes o servicios ambientales. La elección de disponibilidad a pagar en forma directa en bienes y servicios ambientales es una minoría en las posibilidades de los entrevistados, lo cual está directamente relacionado con las condiciones socioeconómicas y culturales de la población, que se relaciona más con la posibilidad de pagar con disposición y dedicación de horas de trabajo.

El instrumento de recolección de información aplicado indagó además sobre la relación entre la existencia de la ciénaga y la posibilidad de conservar sus prácticas culturales. Las respuestas de mayor frecuencia estuvieron relacionadas con la preservación de condiciones productivas y el conocimiento tradicional (Figura 4), lo cual posiblemente evidencia una alta valoración económica de la ciénaga por parte de los encuestados, porque ven una relación directa entre la existencia y conservación del ecosistema y la permanencia del conocimiento tradicional productivo que les permite obtener su bienestar, esto teniendo en cuenta que el ecosistema alberga una gran cantidad de recursos naturales que garantizan el desarrollo de sus técnicas tradicionales de

producción y de vida en general, resultados que concuerda con lo planteado por Aguilera (2006), quien afirma que en la economía ecológica el valor más importante de la naturaleza es el de su propia existencia y el de las funciones que cumple en el mantenimiento de la vida, en los procesos de producción y en la conformación de la cultura y en esta perspectiva, la interacción con otras disciplinas científicas es indispensable para consolidar una teoría del valor, en la que aparte de las cantidades en términos crematísticos, tengan cabida las variables ecológicas y sociales.

\section{Conclusiones}

La aplicación de los métodos de valoración económica ambiental permitió conocer los beneficios que la comunidad percibe o atribuye a la existencia de un ecosistema tan importante como la ciénaga Grande de Beté y los costos que cualquier nivel de intervención implicaría en la disponibilidad de los bienes y servicios ambientales. En este sentido los cálculos mostraron una clara disponibilidad a pagar por usar y conservar no solo los recursos naturales, sino también los aspectos culturales derivados de la existencia del ecosistema.

Se pudo analizar que no solo los bienes de consumo que tienen un precio de mercado, fueron determinantes o significativos a la hora de elegir una disponibilidad a pagar, 


\section{Bioetnia Volumen 10, 2013}

lo que mostró que para las comunidades tanto locales como aquellas que no están asentadas en el municipio del Medio Atrato, su disponibilidad a pagar no está asociada con los costos de uso de bienes de mercado, sino que la valoración de la ciénaga presenta una relación más global, porque asignan un mayor valor a la existencia de la misma más como un recurso natural que es parte integral de sus vidas, que como un valor de uso directo por el conjunto de bienes y servicios que les ofrece hoy. De ahí que la disponibilidad a pagar haya sido mayor para los servicios que para los bienes ambientales y a su vez que esta haya sido positiva independientemente del estado de variables como nivel de ingresos, educación, ocupación, lugar de residencia y edad.

En este sentido, se obtuvieron valores de disponibilidad a pagar de $\$ 23.644,12$ y $\$ 28.977,41$ para bienes y servicios respectivamente, lo que equivale aproximadamente a $\$$ 6.071 '787.860,58 si se tiene en cuenta el espacio muestral tomado para esta investigación (población de Medio Atrato y Quibdó). Estos datos representan más allá de un valor monetario que supla la existencia del ecosistema, la valoración del mismo por parte de la comunidad y expresa su percepción de la importancia de su conservación, lo que aporta una base para la toma de decisiones orientadas al mantenimiento de la ciénaga, teniendo en cuenta las amenazas a las que se encuentra expuesta debido al avance de prácticas extractivas altamente impactantes en la zona, como la explotación maderera y la minería no responsable.

La valoración integral aquí realizada constituye un ejercicio de aproximación al diseño de herramientas que puedan funcionar como incentivos de conservación, que permitan no solo mantener la estabilidad de ecosistemas estratégicos como este, sino la subsistencia, el mejoramiento de la calidad de vida de las comunidades que los han conservado a través de los años y la preservación de las tradiciones culturales que se desarrollan a su alrededor y que tienen un valor incalculable.

Sumado a lo anterior la identificación de los bienes y servicios ambientales prestados por la ciénaga Grande de Beté, a partir de la cual se realizó la valoración integral, constituye adicionalmente la apertura de un abanico de potencialidades de formas de aprovechamiento sostenible y mercados futuros, que pueden ser utilizados en beneficio de las comunidades, de la ciencia y de los recursos naturales, tal es el caso de algunos bienes como potencial genético y productos no maderables del bosque, así como la gran mayoría de servicios ambientales identificados como investigación, turismo, conservación de especies únicas, movilidad y conectividad de especies entre otros, que aunque no poseen mercado actual, conforman un patrimonio con el que cuentan las comunidades y pueden aprovechar a futuro (valor de opción), es decir, una vez conocido y organizado el patrimonio natural, resulta más sencillo incorporarlo de manera ordenada y controlada a los procesos económicos, como parte de la gestión económica de modelos de desarrollo sostenible.

\section{Literatura citada}

Aguilera U. 2006. El valor económico del medio ambiente. [Versión electrónica]. [fecha de consulta: diciembre 10 de 2009]. Revista Ecosistemas 15 (2). Disponible en: http://www.revistaecosistemas.net/articulo

Departamento Administrativo Nacional de Estadística (DANE). 2013. Gran Encuesta Integrada de Hogares (GEIH). Bogotá: DANE-DIMPE-GEIH; $1163 \mathrm{pp}$.

Castiblanco C. 2007. La economía ecológica: una disciplina en busca de autor. Revista Gestión y Ambiente. 10 (3): 7-22.

Cruz G. 2006. Valoración de bienes y recursos ambientales y naturales: El problema de la medición. [fecha de consulta: 11 de diciembre de 2009]. Revista Luna Azul 13. Disponible en: http://lunazul.ucaldas.edu.co/ downloads/0ea3083bRevista13_5.pdf

Gómez-Baggethun E, de Groot R. 2007. Capital natural y funciones de los ecosistemas: explorando las bases ecológicas de la economía. [Fecha de consulta: 20 de diciembre de 2009]. Revista Ecosistemas 16 (3). Disponible en: http://www.revistaecosistemas.net/articulo.asp?Id=496

Instituto de Investigaciones Ambientales del Pacífico (IIAP). 2008. Inventario, priorización y caracterización de ciénagas del Medio Atrato. Informe final. Quibdó: IIAP; 258 pp.

Martínez Alier J, Roca J. 2000. Economía ecológica y política ambiental. México DF: PNUMA, Fondo de Cultura Económica; 52 pp.

Ministerio de Ambiente Vivienda y Desarrollo Territorial (MAVDT). 2003. Metodologías para la valoración económica de bienes, servicios ambientales y recursos naturales. Bogotá: MAVDT; 52 pp.

Naredo JM. 1992. Los cambios en la idea de naturaleza y su incidencia en el pensamiento económico actual. Información Comercial Española. 711: 11-30.

White H. 1984. Asymptotic theory for econometricians. San Diego: Academic Press. 